\title{
Hydraulic servocylinder position control using a fuzzy logic controller
}

\author{
MING-CIIANG SHIH, SIIU-MING LIAW \\ Department of Mechanical Engineering \\ National Chen-Kung University \\ Tainan, Taiwan, 70107, R.O.C.
}

\begin{abstract}
ABSTRAC'T
A fuzzy logic controller, which consists of the linguistic defined fuzzy sets and the control rules, is designed and implemented in a microcomputer to control the position of an electrohydraulic servocylinder. Using the triangle shaped membership function, the normalized universe of discourse, and Tagagi and Sugeno's reasoning for the controller, the position of the servocylinder was successfully controlled.
\end{abstract}

\section{KEYWORDS}

Keywords, Hydraulic Servocylinder, Fuzzy Controller

\section{INTRODUCTION}

The position control of a servohydraulic actuator has been controlled successfully by the application of the modern control theory [1]. Usually, a mathematical model of the servo system is derived and linearized at first, even, determined by using system identification technique; and then, through the system analysis and simulation the controller will be designed. But, it's difficult to achieve the more precision requirement by the conventional controller, owing to the hysteresis, characteristic of the nonlinear flow rate through the valve, the friction force and the uncertainties of the system, e.g. the system temperature, viscosity of the oil , variable mass and force load. The fuzzy logic control concept was described by Zadeh in 1965 . [4]. Instead of the mathematical model, the linguistic variables are used and the relationships of the variables are explainded with fuzzy sets theory. Based on the expert knowledge and experience, the linguistic control strategy can be converted into the automatic control strategy. In the paper, a fuzzy controller is designed and implemented in the microcomputer to control the position of a servocylinder.

\section{EXPERIMENTAL LAYOUT DESCRIPTION}

A schematic diagram of the servohydraulic system is shown in FIGURE 1 , which consists of two symmetrical cylinders, the right one of which acts as a loading generator. The position of the 
cylinder is measured by a linear pulse scale ( model number SM305A, Futaba Co., Japan ). The oil flow to the cylinder is controlled by an electrohydraulic servovalve ( model number SVD-F117.5-11, Yuken Co., Japan ). The load pressure is controlled by a proportional pressure-relief valve. An IBM compatible personal computer PC/XT with a 12 bit DA converter is employed as a controller to excute the control program, and a decoder interface card consists of 2-bit up/down counters and TTLs. A multifunction card consists of programmable peripheral interface device including 8255,8253 and 8279 , which are used to process the position feedback signal and to generate the sampling time to excute a new control loop. The system parameters values are listed in TABLE 1.

\section{FUZZYY CONTROLLER DESIGN}

The block diagram of the structure of fuzzy control on the hydraulic servocylinder control system is shown in the FIGURE 2. The fuzzy logic controlle design include three important steps: fuzzification, fuzzy reasoning and defuzzification. The actual error and velocity signal will be fuzzified with the membership function. The used membership function of error and velocity is triangle shaped, which is shown in FIGURE 3 and can be expressed as the equation,

$$
\mu(x)=(-|x+a|+c) / c, \quad c>0
$$

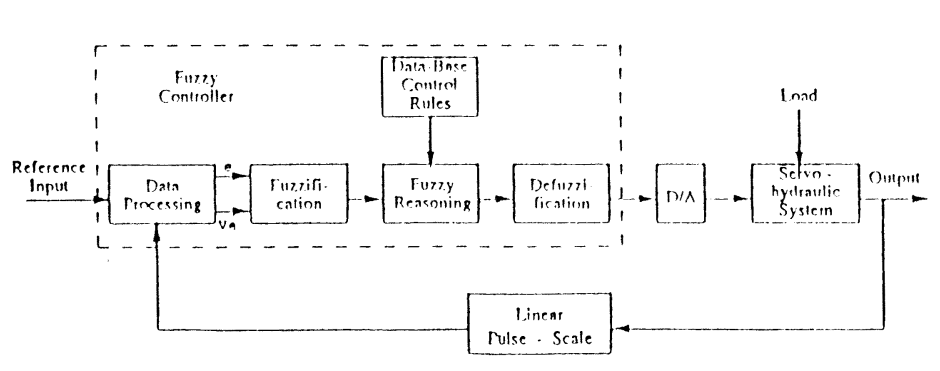

Figure.2 Fuzzy Control Servo-hydraulic System

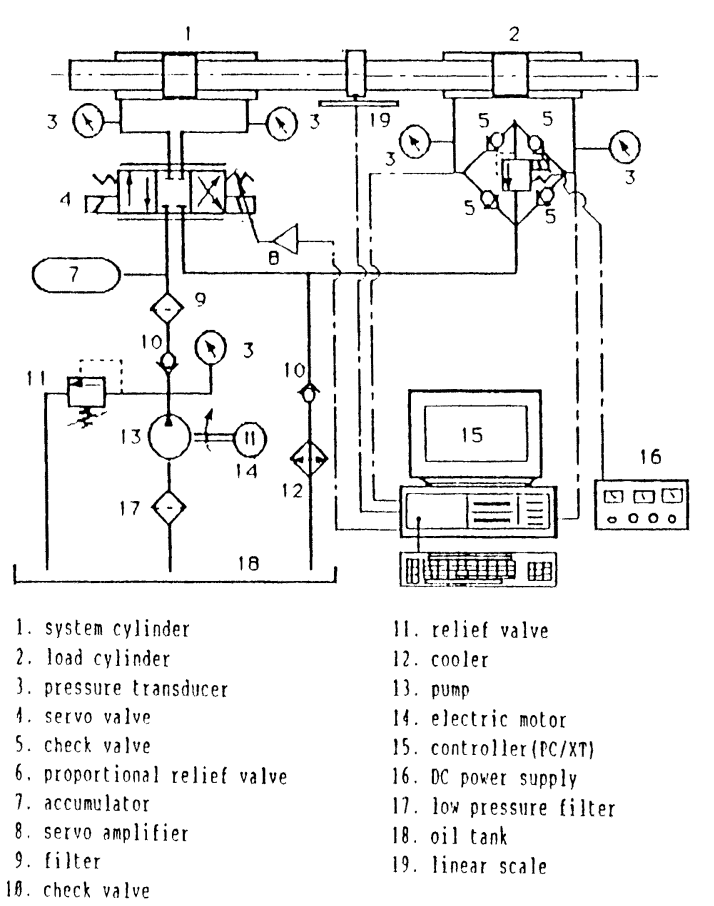

Figure.1 Experimental layout of the servo-

hydraulic cylinder position control system

if $\mathrm{x}=\mathrm{a}, \quad(\mathrm{a})=1$, and the parameter $\mathrm{c}$ represents the wide or narrow grade of the membership function. The process of the fuzzy reasoning is based on the data knowledge and the control rules the control rules can be defined and obtained from the expert experience and engineer's knowledge, learning and the operator's control action. In the article, we developed the control rules for the hydraulic servocylinder position control with Tagagi and Sugeno's reasoning method [6] :

$$
\begin{gathered}
R^{m}: \text { if } x_{1} \text { is } A_{m 1} \text { and } x_{2} \text { is } A_{m 2}, \ldots, x_{n} \text { is } A_{n n} \text {, then } \\
u_{i}=f_{i}\left(x_{1}, x_{2}, \ldots, x_{n}\right)=a_{1} x_{1}+a_{2} x_{2}+\ldots+a_{n} x_{n}
\end{gathered}
$$

\begin{tabular}{|c|c|}
\hline Parameter & Vulue \\
\hline Cross Section Area of Piston (cm) & 30 \\
\hline Stroke of Cylinder $(\mathrm{cm})$ & \pm 15 \\
\hline Total Mass of Cylinder and Rod $(\mathrm{kg})$ & 30 \\
\hline Supply Pressure (bar) & 100 \\
\hline Time Constant of Servovalve (ms) & 8 \\
\hline
\end{tabular}

Table 1 Parameter of the controlled system 

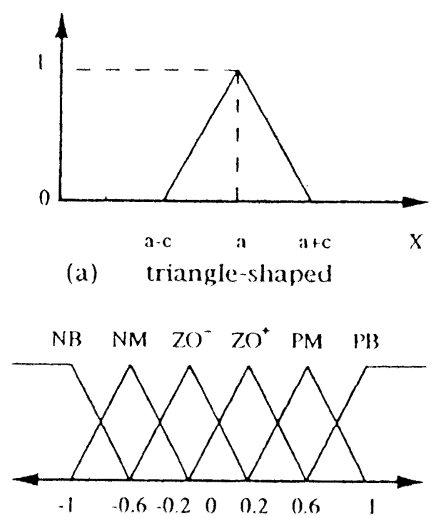

(b) error

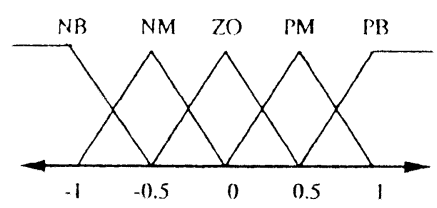

(c) velocity

Figure.3 Membership function of error and velocity

For the design process of the fuzzy controller are as follows:

1. Choice of the state variables and the membership functions normalized

For the fine regulating, the larger the number of the fuzzy state variables, the more control rules will be. But the control rules will be more complex and difficult to design. To reduce the number of the control rules and to achieve the good control performace are important for the fuzzy controller design. In the article, the error between reference input and system output and the velocity are chosen the state variables, which gain are GE and GCE, separately. There are six fuzzy sets defined for the error: Positive Big (PB), Positive medium $(\mathrm{PM})$, Zero Plus $\left(\mathrm{ZO}^{+}\right)$, Zero Minus $\left(\mathrm{ZO}^{-}\right)$, Negative Medium (NM) and Negative Big (NB); and five fuzzy sets for the velocity: Positive Big (PB), Positive medium (PM), Zero ( $\mathrm{ZO})$, Negative medium (NM) and Negative Big (NB).
2. Determine the control rules

The fuzzy control rules are decided as follows :

$\mathrm{R}^{1}$ : if $\mathrm{e}$ is $\mathrm{PB}$, then $\mathrm{u}_{1}=\mathrm{p} \cdot \mathrm{e}$

$\mathrm{R}^{2}$ : if $\mathrm{e}$ is $P M$, then $\mathrm{u}_{2}=\mathrm{p} \cdot \mathrm{e}$

$\mathrm{R}^{3}$ : if $\mathrm{e}$ is $\mathrm{ZO}^{+}$and $\mathrm{Ve}$ is $\mathrm{PB}$

then $\mathrm{u}_{3}=\mathrm{p} \cdot \mathrm{e}+\mathrm{q}_{1} \cdot \mathrm{Ve}$

$\mathrm{R}^{4}$ : if $\mathrm{e}$ is $\mathrm{ZO}^{+}$and $\mathrm{Ve}$ is $\mathrm{PM}$

then $\mathrm{u}_{4}=\mathrm{p} \cdot \mathrm{e}+\mathrm{q}_{1} \cdot \mathrm{Ve}$

$\mathrm{R}^{5}$ : if $\mathrm{e}$ is $\mathrm{ZO}^{+}$and $\mathrm{Ve}$ is $\mathrm{ZO}$

then $u_{5}=p \cdot e+q_{1} \cdot V e$

$\mathrm{R}^{6}$ : if $\mathrm{e}$ is $\mathrm{ZO}^{-}$and $\mathrm{Ve}$ is $\mathrm{ZO}$

then $u_{6}=p \cdot e+q_{2} \cdot v e$

$\mathrm{R}^{7}$ : if $\mathrm{e}$ is $\mathrm{ZO}^{-}$and $\mathrm{Ve}$ is $\mathrm{NM}$

then $u_{7}=p \cdot e+q_{2} \cdot V e$

$\mathrm{R}^{8}$ : if $e$ is $\mathrm{ZO}^{-}$and $\mathrm{Ve}$ is $\mathrm{NB}$

then $\mathrm{u}_{8}=\mathrm{p} \cdot \mathrm{e}+\mathrm{q}_{2} \cdot \mathrm{Ve}$

$\mathrm{R}^{9}$ : if $\mathrm{e}$ is $\mathrm{NM}$, then $\mathrm{u}_{9}=\mathrm{p} \cdot \mathrm{e}$

$\mathrm{R}^{10}$ : if $\mathrm{e}$ is $\mathrm{NB}$, then $\mathrm{u}_{10}=\mathrm{p} \cdot \mathrm{e}$

In the control rules 1 and 2, the error is considered only, because the error is big or medium, the control signal must be large in order to reduce the rise time. For the control rules 3,4 and 5 , the actual output value is approaching to the command value, the error becomes smaller and the velocity term will affect the overhoot of the system. Using the weighting factors $p$ and $q$, then the control signal $u$ can be regulated and make system response faster and no overshoot.

If the error is zero plus, the weighting parameter is $q$, but, if the error is zero minus, the parameter will be changed to be $\mathrm{q}$. For the fuzzy reasoning process, the grade of the membership functionW , can be written as 
$\mathrm{Wi}=\prod_{\mathrm{j}=1}^{n} \mu_{\mathrm{A}_{\mathrm{ij}}}\left(\mathrm{x}_{\mathrm{j}}\right)$

$\mathrm{i}=1,2, \ldots . .10$, and $\mathrm{n}$ is the number of variables,

$x_{1}$ and $x_{2}$ are the two state variables error $e$ and

velocity $V_{c}$ in the study.

3. Defuzzify the control signal

Through the defuzzyfication process, the control signal u to the servocylindercan be obtained by the following equation :

$$
\mathrm{u}=\frac{\sum_{\mathrm{i}=1}^{10} \mathrm{~W}_{\mathrm{i}} \mathrm{u}_{\mathrm{i}}}{\sum_{\mathrm{i}=1}^{10} \mathrm{~W}_{\mathrm{i}}}
$$

The above controller design process can be summarized as the flow chart, in FIGURE 4.

\section{EXPERIMENTAL RESULTS}

In the experimental study, the supply pressure and the sampling time are cho-

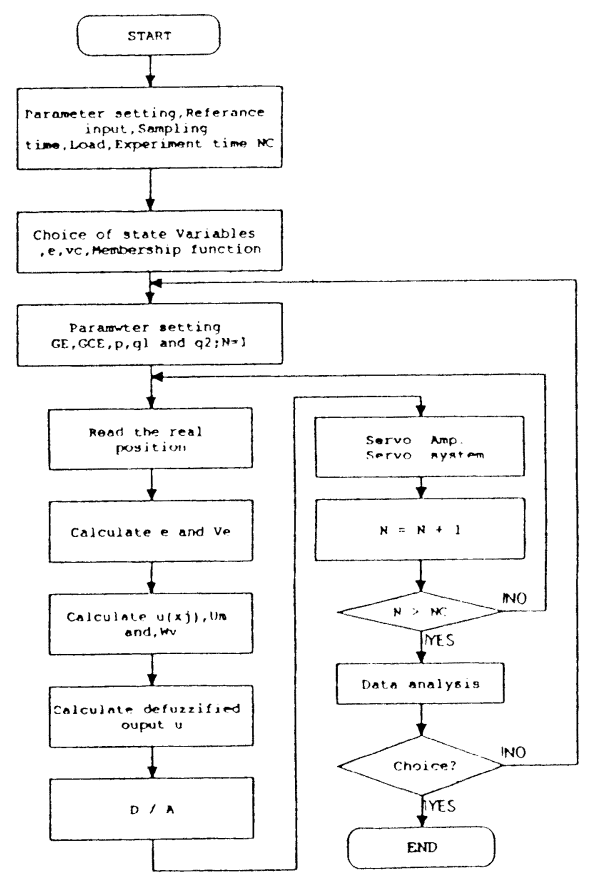

Figure.4 Flow chart of fuzzy control system sen as 100 bar and $10 \mathrm{~ms}$, respectively.

\section{a. STEP INPUT WITHOUT EXTERNAL IOAD}

Through the try and error design process of the fuzzy controller, the scaling factors GE and GCE are equal to 0.5 and 0.2 , and the parameters $p$, $\mathrm{q}_{1}$ and $\mathrm{q}_{2}$ are equal to 5, -0.2 and 0.2 , respectively.

FIGURE 5 shows the time response of the system without external load. In fig.5(a) and (b), one can see that the velocity of the cylinder is almost constant from the start point till the command value. At the near of the command signal, the velocity is suddenly reduced to zero, and then the position hold constant. From fig.5(c), one can understand that the control signal is saturated at first, and then reduced to zero from $0.74 \mathrm{sec}$ till $1.20 \mathrm{sec}$, after that time the control signal is zero.

Although the system is excuted without external load, the loading pressure isn't equal to zero because of the friction force of the cylinder.

\section{b. The System Under Variable Load}

FIGURE 6 and 7 have shown the time responses of the system under periodic variable load pressure, the former load pressure from 20 bar to 40 bar till 1.7 sec and the latter from 30 bar to 60 bar

till 2 sec. Owing to the passive load generator unit, the load is the friction force only at the steady state. Comparing the experimental results from FIGURE 6 and 7 , one can see that the cylinder under larger variable load reached the command value later and the velocity of the cylinder is slower apparently, besides, both of the two responses have no overshoot. The loading effect is relatively small by using the fuzzy logic controller. 
In the FIGURE 8, the time responses of From the above experimental results, the system with different input signals one can make the conclusions as follows: are shown. With larger reference input, it takes longer time for the cylinder to 1 . The application of the fuzzy control reach the command position, the control technique on the hydraulic servocylisignal is also longer at the saturated ndrer position control is easy to get a level. Besides, owing to the characteristic very satisfactory performance, including of the load pressure relief valve, the the fast response, no overshoot and load pressure oscillates till the cylinder small steady state errors. reaches the command position.
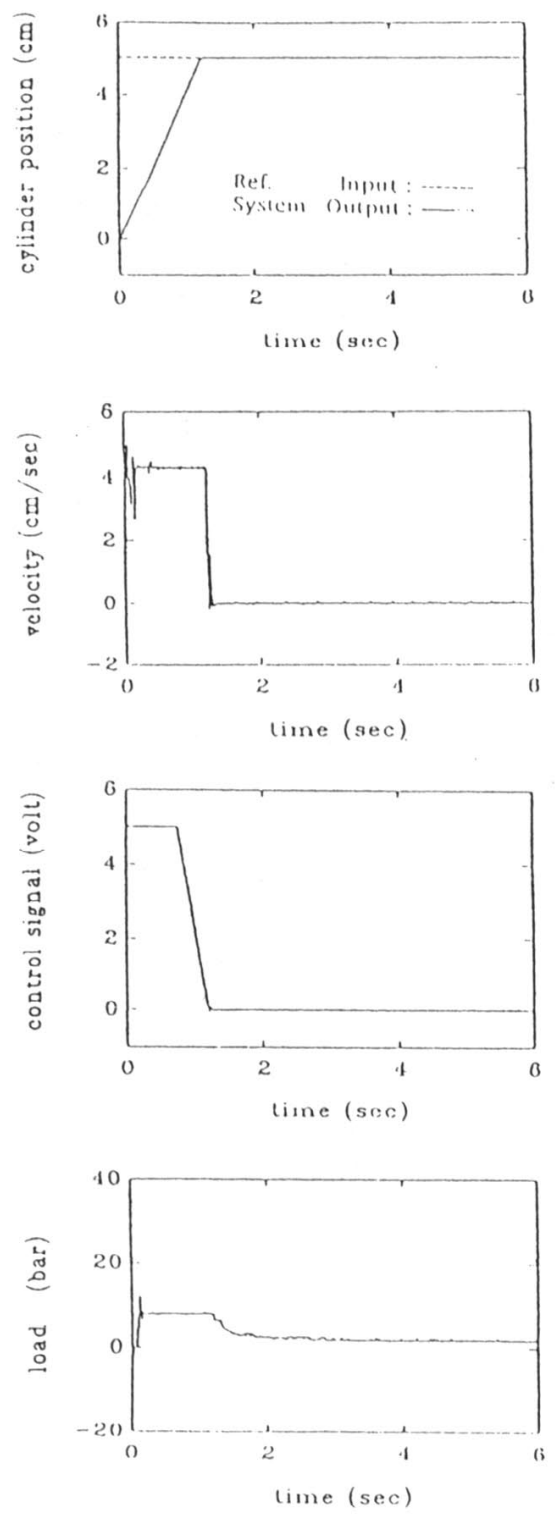

Figure.5 Dynamic responses of the fuzzy control with reference input $5 \mathrm{~cm}$
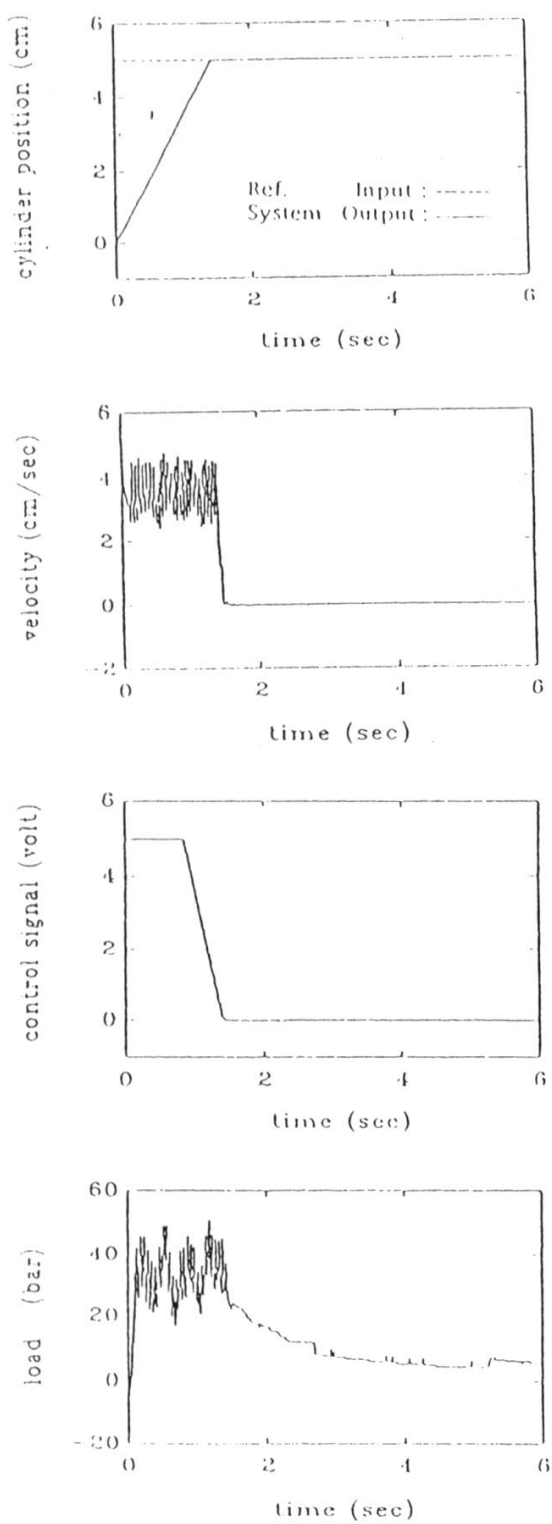

Figure.6 Dynamic responses of the fuzzy control with medium variable load and disturbance
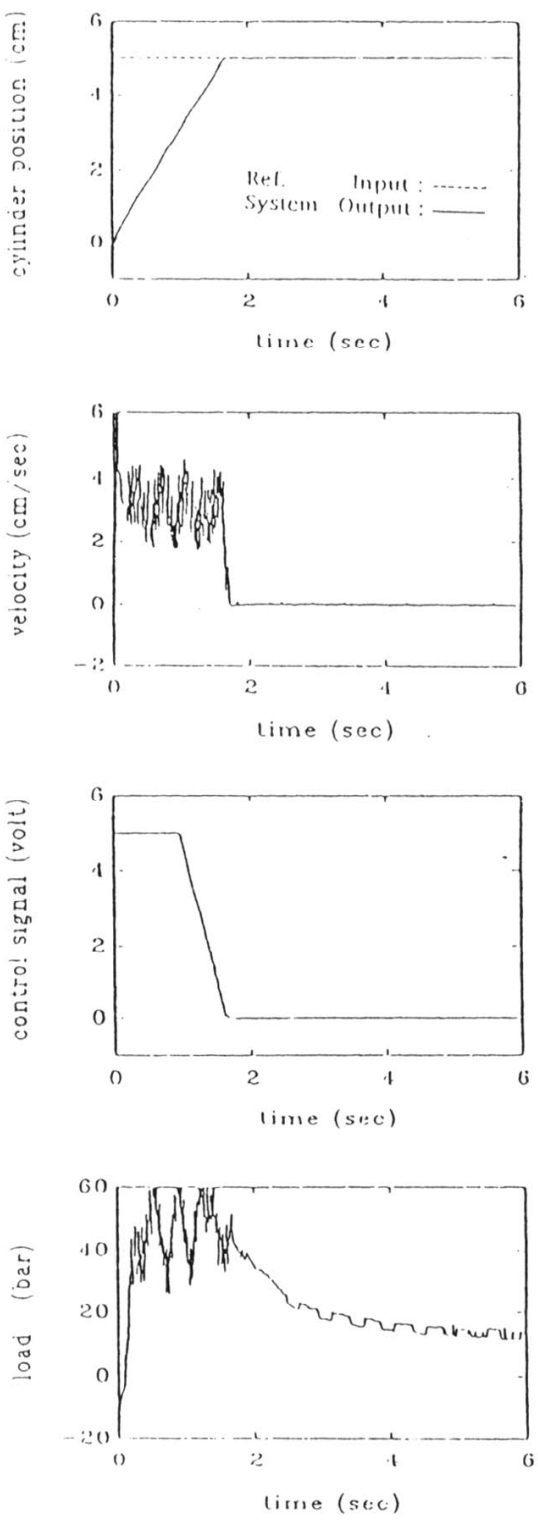

Figure.7 Dynamic responses of the fuzzy control with large variable load and disturbance 

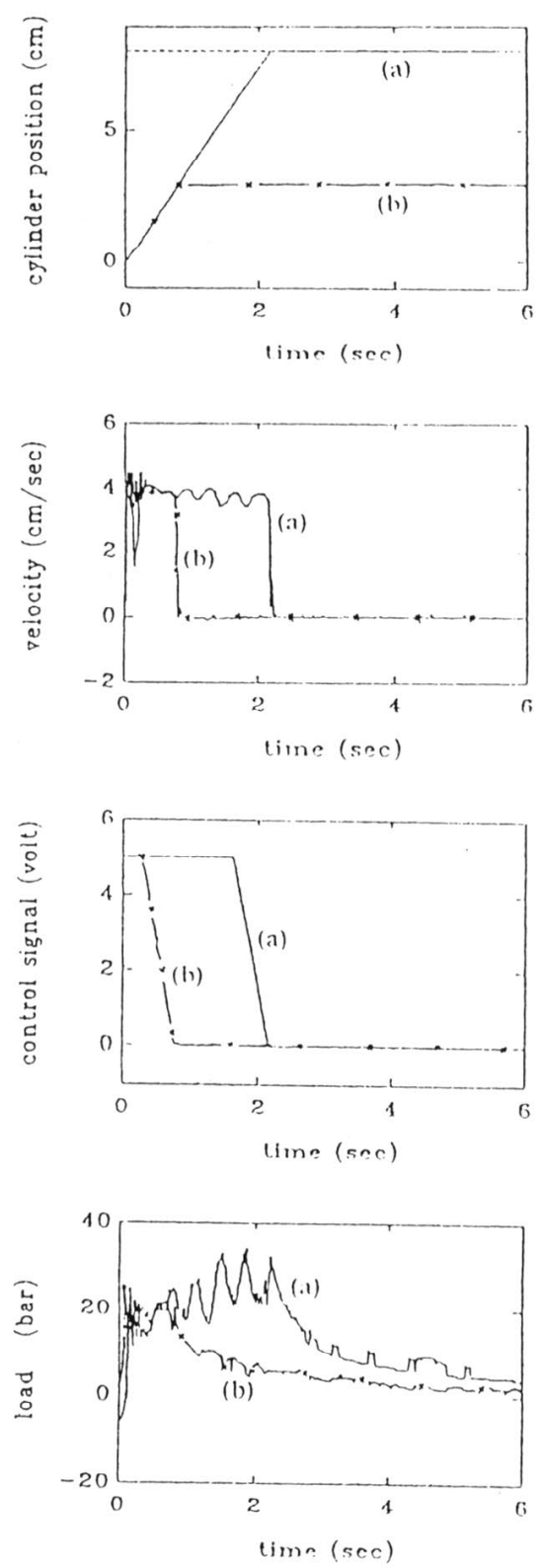

Figure. 8 Dynamic responses of the fuzzy control with reference input (a) $8 \mathrm{~cm}$ (b) $3 \mathrm{~cm}$
2. The control rules are reduced and simplified to ten rules in the study. Thecomputation time is also relatively reduced and the system has good robustness performance - variable load effect rejection and low sensitivity to the system parameter changes.

\section{REFERENCES}

1. W. Backe', " Servohydraulik ", Vorlesungsumdruck of TH Aachen,Germany, (1992)

2. T. Virvalo, H. Koskinen, " Fuzzy logic controller for hydraulic drives ", 10th, Aachener Fluidtechrisches Kolloguium, Band 2, pp.225-240, 1992.

3. Pietola, M. T. and Vilenius, M. J." Theoretical and experimental study of the effect of varying load on the dynamics of a P, MRAC or P+PID controller electrohydraulic position servosystem", Prc. Instn. Mech. Engrs., Vol. 203, part c, pp. 267,1989

4. L. A. Zadeh, " Fuzzy sets ", Information and Control, Vol. 8, pp.338-353, 1965

5. M. C. SHIH and P. C. CHEN, " An Experimental study on the position Control of a Hydraulic Cylinder using a Fuzzy Logic Control ", JSME International Journal, Vol. 34, No.4,pp.481-489,1991

6. C. C. Lee, " Fuzzy logic in control systems: Fuzzy logic controller, part I ", IEEE Trans. Systems, Man, and Cybernetics, Vol. 20, No.2, pp.408-418, 1990

7. C. C. Lee, " Fuzzy logic in control systems: I'uzzy logic controller, part II", IEEE Trans. System, Man, and Cybernetics, Vol. 20, No.2, pp.419-435, 1990

8. X. T. Peng, "Generation Rules for Fuzzy Logic Controller by Functions ", Fuzzy Sets and Systems, Vol.36, pp.83-89, 1990 